\title{
About motivated project teams, user expectations, proof-of-concept testing and the after-a-good-project-hang-over.
}

Piet Verhoeve (1), Ann Ackaert (2), Jan Van Ooteghem (2), Jeroen Hoebeke (2), Maarten Steenhuyse (2), An Jacobs (3), Annelies Veys (3), Mieke Van Gils (4), Heidi Buysse (5), Stijn Agten (6), Brenda Aendekerk (7), Rina Vangerven (7)

(1) Televic N.V. p.verhoeve@televic.com, (2) IBBT - UGent/IBCN, Belgium, 32933149 35, ann.ackaert@intec.ugent.be; (3) IBBT - VUB/SMIT, Belgium, (4) IBBT - K.U.Leuven, Belgium, (5) IBBT - UGent/MIG, Belgium, (6) IBBT - UHasselt/EDM, (7)Wit Gele Kruis VZW

The sense of making good use of ICT applications in support of the activities of the daily live (ADL) of elderly people seems all too obvious. Meanwhile time adoption of these new services and applications in the healthcare market is slow, extremely slow in comparison with other sectors. Careful and user-centered design, proof-of concept testing and intelligent value market analysis, seem not to be sufficient to continue the life of many applications after running-out of project subsidies.

This chapter will describe the hands-on-experience and the lessons learned of a multi-disciplinary team in Belgium, who has elaborated from 2005 to 2009 two R\&D projects in the field of eHomeCare services (respectively COPLINTHO(1) and TranseCare(2)). These projects involved research teams from different disciplines and with industrial and care organizations as project partners. The projects were co-funded by the care organizations, industrial partners and the Flemish IWT and IBBT subsidiary channels. Currently efforts are being made both by the involved companies and by the IBBT to bring some of the developed services to the market. How to cross this gap from projects to market is still a challenge in the eHomeCare area

\section{Description of the project}

In both cases the project consortium consisted of a multi disciplinary team of academic, non-profit and commercial organizations covering technical (academic $\&$ commercial), user (academic), legal (academic) and healthcare (non-profit) expertise domains. Both projects also aimed at putting co-development mechanisms 
into practice in order to enhance the co-operation between the industry sectors involved, the potential users, the non-technical and technical research groups. Each project was finalized by realizing a 'proof-of-concept' demonstration during the second project year in order to illustrate in a live-environment the concepts developed for an innovative communication platform for the eHomeCare sector. The exact configuration and functionality of such a proof-of-concept demonstrator was each time defined by the end of the first project year.

The main objective of the COPLINTHO project was to study the introduction of ICT in the area of the home care (denoted as eHomeCare). Both technological and non-technological issues were taken into account in this project. The nontechnical study covered a wide range of aspects such as regulation, liability, social impact and usability of the services (both for the caregiver as for the patient). The technical part of the project was devoted to research and development of several innovative ideas for the eHomeCare environment such as the creation of an ambient intelligent environment to support the care process of the patient, the creation of innovative communication platforms tailored to the needs of patients, nurses or homecare in general and the creation of secure and efficient data exchange services between all involved actors. In short, the COPLINTHO project aimed at bringing ICT to the homecare environment, more specifically oriented towards diabetes and multiple sclerosis patients.

The second project "TranseCare" narrowed down the scope towards people suffering from chronic diseases and/or from degenerative disabilities due to age. Although specific pathologies were encountered during the field tests (i.e. actual persons), the methodologies and concepts were developed based on the more generic categories such as chronic diseases and degenerative disabilities irrespectively of the age at which they occur. The objective of the TranseCare platform was to support these people through the aid of an ICT platform, focusing on real needs, and real life situations. The TranseCare project aimed at taking the concept of "independent living systems" a step further than mere R\&D conceptual demonstrators. In short, the TranseCare project aimed at adapting the ICT towards the users instead of the other way round.

The project focused on three themes:

(i) The creation of a "transparent home environment", offering to users an "open window" on the world in their home environment through the aid of ICT

- An easy to reach "point of contact/central desk" supported with interactive video allows emergency or social calls (e.g. peer groups or communities)

- The study and development of game concepts integrated in this interactive video platform (occupational therapy, infotainment). 
- Study and exploitation of the concept of virtual buddies. Stimulate social contact (with e.g. the "good morning-good evening service), decrease the feeling of isolation

(ii) The creation of a "transparent data exchange platform", offering the adequate information to both professional and family caregivers.

- The study and definition of data exchange flows for the required settings(medical, care).

- Semantic interoperability, both at the level of syntax or at the level of true information content.

- Study and development of all necessary architectural components for the creation of a web-service based data exchange platform (privacy and security issues, authentication, authorization, advanced engines for dynamic workflow organization, development of a Patient Information Location Service).

- Study of all related legal and liability issues.

(iii) The creation of a transparent network environment offering support to both domotica and mobility aspects

- Adaptation of Personal Network (PN) (self configuring and self organizing heterogeneous networks) concepts to the involved care settings.

- Use of proxy technology assessment techniques to collect and analyze user feedback.

- Research on PN-federation (the collaboration between PN's) to increase collaboration in between actors (e.g. patient and nurse).

- Study of all legal and privacy issues in this domain.

The result of both projects was a custom configurable eCare platform that connects to the users" television through the Internet, and allows for video-telephony with a professional care help-desk, other users of the eCare system and informal caregivers using an instant messaging client on their PC.

\section{Development of the project}

\subsection{Arguments to start the projects}

The major arguments for these projects were the societal challenges that are faced in the near future due to the ageing society. Indeed, already today there is a global shortage of qualified care staff, hospital and nursing beds which cannot only be solved by building more infrastructure or training additional persons in healthcare domains. Based on population numbers and predictions towards the fu- 
ture (3), the ratio of active persons compared to the care needing persons will drastically shift in the next decades; a ratio which has practically remained constant over the past century as indicated in the figure below. This figure shows the numbers for Belgium as an example, but they are applicable to each country in the western world. If the present model of care would continue, about half to twothirds of the active population needs to be active in the healthcare sector by the year 2025 .

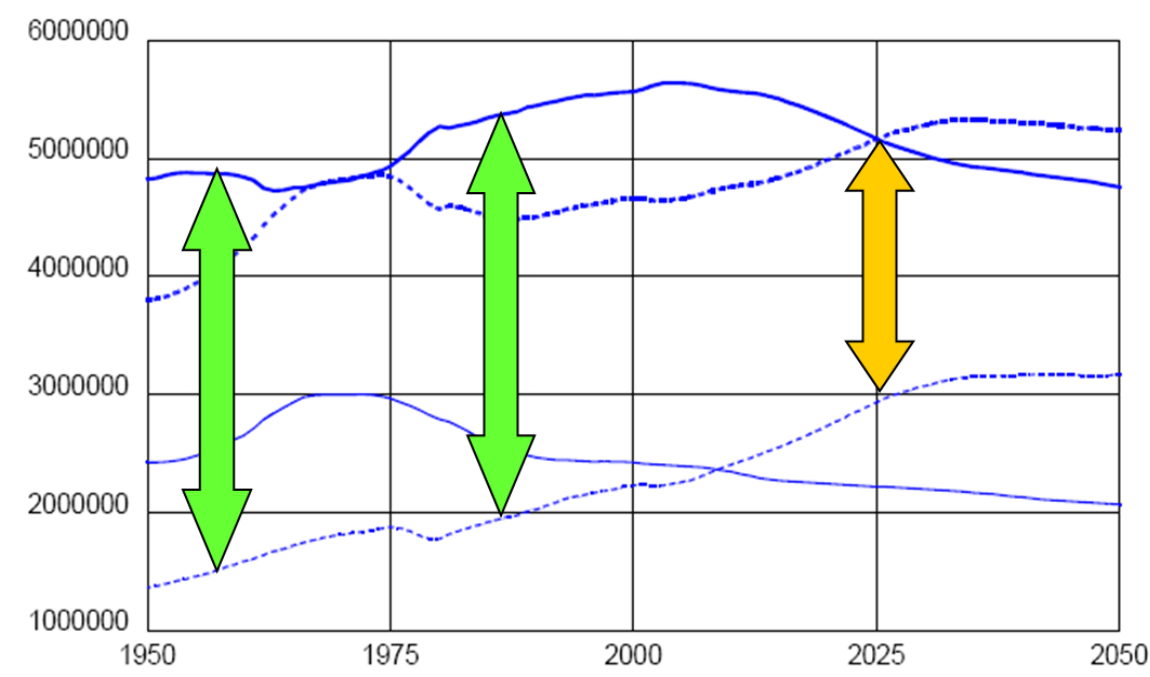

Figure 1: evolution of the population in Belgium (legend anno 1950:from top to bottom respectively age groups: 20-59 / dependent eldery an youngsters / 0-19 / $60+)$

While it is clear that not all elderly persons will be able to find a place in a nursing home, another societal change is taking place: both elders and society are more and more aware of the fact that most persons like to continue living at home continuing their independent lifestyle with specific care support provided at their location by professional nurse and family caregivers. Although this general user trend can be considered as a clear answer to the shortage in nursing homes, it aggravates the shortage of nurses since a home nurse also spends a lot of time traveling from one patient to another in case of homecare.

It should be clear that a change in the caring process will be part of the near future in order to be able to provide sufficient care for society. Although changing the care system is not part of the COPLINTHO neither the TranseCare project, it should be left to the healthcare professionals and governments, the multidisciplinary team was (and is) convinced that ICT can provide a supporting key role in making the healthcare process more efficient, providing more caring time for the nurses by reducing time spent on non-care activities and enabling communication 
process to exchange information electronically vs. paper based solutions. Examples of such efficiency enables are:

- a better/faster communication between all actors involved in the care process; e.g. a home nurse stays up-to-date on the fact that a patient is admitted to the hospital so that she doesn't need to visit the patient at home, that the meals-on-wheels service is informed, etc.

- video based communication that can be used to build a trusting relationship with a coordinating nurse without the need for that coordinating nurse to drive from home to home each time. In addition the video communication will give the nurse a better idea of the patient's condition compared to an audio only (phone) based scenario.

- Secure electronic communication between professional and nonprofessional caregivers.

\subsection{What were the goals / expectations}

As indicated in the previous section, the combined expectation of all partners was to gain a better insight and to advance the introduction of ICT technologies into the home care sector. This global expectation can be split into different more concrete goals, based on de different axes of the multidisciplinary team:

\section{The service axis (care \& user):}

The addition of telecommunication to the offered PAS-service (4) might possibly be of great value for the nurse/operator of the alarm central. With help of the camera, possibly by non-verbal communication, they are able to check on the patients room and make a better estimation of the patient situation. In this case the unnecessary call for help can be avoided (and vice versa).

The user expects a simple implementation with an ease to use and a flexible programming of the camera. Is this generation ready for this? When selecting the patients, we see motivated caregivers, however the user group (elderly) tends to reject the implementation of the technology (e.g. due to stigmatic effects), how can this introduction hurdle be circumvented?

Improving communication with the social networks of the users: not only on personal level (family and friends) but also care specific (medical staff, caregivers, coordination nurses, etc.)

\section{The technological axis}

The nurse and operator of the alarm central request a clear speak-listen connection, a good image quality, the possibility of the implementation of the patient file and the history of the calls. These items are needed to make a good estimation of the call which will be followed by the correct interventions. 
Concerning our target group, we should be able to modify the program on request/demand since elderly users need specific, adapted offerings according to their needs in their specific condition which evolves over time.

Technologies should be based on open standards that the solution can make use of available networks such as internet connections and can integrate with existing systems in order to provide a smooth adoption path rather than requiring a big bang style conversion. In addition providing a system without explicitly creating a lock-in scenario is being preferred by most organizations (both profit and non-profit).

The projects aimed at implementing a data and information pulltechnology based scenario from home care or hospital with specific access rights (social nurse, nurse, etc.) for all users. This had to be done by demonstrating actual electronic exchange of necessary data between hospital and home care in a secure and trustworthy style such that all users (both providers and consumers of information) will be inclined to use the systems.

\section{The business axis}

eHomeCare comprises a large number of actors, with not only different interests and motivations but also very different financial models (government, non-profit, commercial, personal relationship, etc.). Concerning the cost efficiency, video communication might serve as a filter at the alarm centres, thus providing a benefit for the care organization. However we can wonder if the telecom operators are ready concerning the financial part of lowering the costs of the connection fees and monthly rent.

Business modelling based on financial flows typically ends in very complex figures and dead end reasoning. Can a more simple framework be devised which will enable the introduction of ICT in home care environment.

\subsection{What were the innovative elements?}

The projects encompassed several innovative elements on the different axes of the multidisciplinary approach. They can be summarized as follows:

\section{Multidisciplinary axis}

As both projects encompassed a multidisciplinary team, the goals and expectations of each partner (stakeholder) were oriented in different directions. During the first project COPLINTHO, this proved to be a tedious process resulting in delays and complications to realize the demonstrator; the project even had to be extended in order to have a minimal field testing period. During the execution of the TranseCare project a specific "white book" methodology(5) has be devised based on the lessons learned during the COPLINTHO project. This approach not only allowed the different stakeholders to express their goals but also proved to be an ef- 
ficient method that facilitated the discussions between technical and non technical participants. Furthermore it enabled the projects to create a clear focus on the use cases, the user scenarios and the demonstrator from the early start of the project while allowing to adapt the specific conditions of the demonstrators according to the outcomes of the technological research tracks (which are by nature uncertain at the start of a research project). This "white book approach" consisted of first distilling specific user scenarios based on the goals and expectations of all partners, using the persona methodology (6-8). Secondly the approach added a technological mapping to each scenario, which encompassed the relevant technological components and communication channels between the devices. In doing so, it was possible to estimate the technological risks of each user scenario according with respect to actually building the demonstrator. Furthermore this approach also allowed the non-technical partners to take an active role in the discussions as each technological setting was specifically linked to each user scenario. The result of this approach was that already in an early stage a clear consensus was created which classified the user scenarios and demonstrators to "field", "lab" or "concept" categories, thus managing the expectations of all partners, lowering the implementation risks in the field, and increasing the chances towards success at the end of the project.

\section{Technological axis}

The communication platform studied en developed in the projects was not just about bringing the video communication to the end user, but it was about creating a solution that is fit for care. This means that the solution had to match a set of challenging requirements with respect to quality, safety, security, possibility to install (by non technical staff), usability (by non tech savvy elders) and the legal framework (privacy \& trust issues). During the sequential projects, the concept evolved from a healthcare terminal based design to the far less intrusive design of Xtramira $^{\mathrm{TM}}(9)$, which connects to the television set of the end user. The multidisciplinary research helped to create the innovative design of the user interface allowing for a practically manual free usage (by the elderly), while at the same time realizing a high trust factor concerning privacy issues ("can I be seen on the internet").

The Virtual Private Ad-Hoc Network (VPAN) (10) concept aims to securely and automatically interconnect distributed groups of devices. It aims to create secure self-organizing virtual networks in which any service can be deployed, shielded from the outside world. Since the services we want to provide involve different players (elderly, caregivers, family...), the interconnection of their devices in a user friendly and secure way is a key feature. Through this VPAN technology, multiple devices at distant locations that need easy access to each other's data and services are grouped in a logical, virtual, network. This is done automatically and securely, regardless of the heterogeneity of underlying networks. Based on security information, devices can verify if they are member of the same group, after which they can setup secure links to exchange information. The network will 
organize itself regardless of the location of devices and any movement of devices will trigger a reorganization without user interaction.

The technology also contains a service framework which automatically announces the availability of services to other members of the same group and allows user friendly access and management. Any network based service can be deployed on top of the logical network formed by the members of the group. As such, the VPAN technology effectively hides the technological complexity of the interconnection between devices and creates a permanent and closed group. Furthermore, the technology meets the flexibility requirements. Not only in terms of devices and services, but a device can also be member of multiple groups, which are all nicely shielded from each other.

The web based video enabled central desk can also be considered as a technological innovation. A new framework was developed that could realize a fully zero install (i.e. easier roll out conditions) application for the alarm centre central desk functionality. This application did not only implement audio and video but also realized a queuing/dispatching functionality for directing the alarm calls to the available staff. Additionally the application also encompassed a remote video camera activation toolset which complies to the legal requirement in the elderly homecare setting. Finally the application integrated a case management portal.

\section{Care axis}

The innovation according to the care axis is the integration of telecommunication within the first line aid to develop supportive technology for caregivers, the nurse and other first line aid operators. This means that a better estimation of the problem can be made by telecommunication means. The technology created in these projects serves to guard the security of the user, taking into account the protection of the privacy of all users involved yet still allowing for efficient information exchanges to support the care process.

As was demonstrated by A. Arnaert (11), these projects also demonstrated that the use of video technology can increase the self esteem, lifestyle, well being and the participation of the patient to the society. In addition the results of both projects have indicated that this also applies for the family caregiver. Thanks to the ICT technology, more frequent (shorter) communications can be realized, hence providing a better understanding of the patient's condition and life, which results in a more comfortable feeling.

\section{Business axis}

As indicated above, business modelling based on financial flows typically ends in very complex figures and dead end reasoning. The number of published value network models to evaluate the economic feasibility of eCare services is limited. A literature study and expert interviews have been executed. Studies on stakeholder readiness analysis (12) as well as a review of guidelines (13) and key indicators (14) for cost-benefit analysis (15) for telehomecare could be found, but are rare. During the projects, a multi-actor analysis indicates the benefits for all actors 
involved in the eCare ecosystem. The resulting mathematical model will help formulating a business case towards governmental agencies, insurance companies, healthcare providers or private investors as to how much and how soon to invest in eCare. $(16,17)$

\section{Outcome of the project}

\subsection{How and why did the project succeed / fail}

To measure the success of this we make a distinction between the experiences for the user groups and the experiences with the multidisciplinary project team and technology itself.

\section{Multi disciplinary team}

A success is definitely the cooperation and communication with the technology partners. Healthcare received a very good flow of information and support of the multidisciplinary team.

\section{User axis}

In healthcare we need to make a distinction between 4 user groups: the elderly, his/her caregivers, nurses, and the operators of the alarm central since their expectations are different.

The operators of the alarm central were a little anxious at the start. They were fearing an increase of workload. A disappointing result was the patient enrolment for the TranseCare-study. For the test case of video surveillance only one patient met the enrolment criteria and gave consent. The inclusion criteria were very challenging and requested a single-living patient of the "Wit-Gele Kruis" organisation with a mobility score of 2 or more and a possibility of a toilet-visit score of 2 or more on the Katz and Weckx scale. A second reason for the low inclusion rate was due to the fear of the elderly for new technology. (see Section 6.1) The elderly person that was included, was very enthusiast at start. It should be remarked that we do not know whether this was due to the extra visits or due to the technology. She suffered from dementia, but had still good cognitive skills.

To search for test patients, one of the departments of the "Wit-Gele kruis" organisation was contacted. They were requested to "spread the word" and search between their patients for patients fulfilling the inclusion criteria. Although, at start they were very scared of the technology (see also Section 6.4), the nurse of the test person was very happy she tried out the technology; she gained confidence to use this type of applications. This is a success on a small scale since we first need a convinced nurse before to convince the patient. 
Although the caregivers of the test person were living at a short distance from their mother, they were very enthusiastic about the technology. They were excited to see their mother (in-law) on a screen and have more contact with her without the explicit need to frequently drop by in an unannounced style. The net result of this additional contact was that the caregiver was more at ease that everything was all right. Can we generalize this result? Can we conclude that this application is a success for the caregivers?

Although the technology components developed proved to be operational and successful, the dependence of eHomecare on external companies like for example the internet provider proved to created difficulties for testing. Not only the prices were not adapted, also when there was a connectivity issue it took too long before to find the problem (due to the many actors involved and the variability in modem install base) and solve it. Shortening problem solving times would make it is easier to retain test persons. However when the users are asked whether they would like to adopt the technology, the price of the technology and accessories is very important (18).

From user point of view, we have the impression that the technology would be a bigger success and easier acceptable if it would be provided by packages (everything needed included); however even research based on prototypes, as in our cases, can provide valuable feedback for further and future product development. Another item to take into account is the fact that technology should be that simple that in case there is a small problem (connection issues etc) the nurse can solve it.

\section{Technological axis}

At the end of the COPLINTHO project, the terminal based demonstrator was at the same time successful and a failure. It was successfully designed, operational and proved to be an excellent demonstration that the multidisciplinary team could deliver actual results. Furthermore it proved to be a good "ambassador" into the market place, resulting in a steep climb of the reputation of Televic N.V. previously active in residential healthcare but not active and not known in the home care market. It was a failure due the fact that the user validation of the terminal proved to be not yet at the level of the end user skills (suffered from multiple sclerosis disease), and that it proved to be to stigmatic (having a healthcare terminal indicates that the user is dependent). Both aspects were considered as valuable lessons learnt for the second project TranseCare.

During the TranseCare project, the homecare vision was elaborated and a new concept was devised that focused on the non-intrusiveness and an elderly-proof usability without ending up an a stigmatic situation (neither towards the care dependency nor towards the especially-for-the-old label). This approach resulted in a device that combined the correct functionalities (social calling, alarming function and telemedicine) while being easy to use for the elderly and non-stigmatic at an achievable pricing rate for the target market (compared to existing non-video capable products). The device is currently commercially available under the name Xtramira $^{\mathrm{TM}}$. 
The VPAN concept was successfully realized the whole set of specific requirements. It can be argued that they are or can also be solved by other existing protocols. However, these solutions only tackle one specific aspect, but fail to tackle the other requirements. Simply combining a number of these solutions is also too limiting in order to meet all requirements. This is exactly the strength of the VPAN concept, that it is an integrated solution that meets all requirements.

During the TranseCare project, the VPAN technology has been used to realize an end user scenario in a residential care setting at the "OCMW De Vijvers" organisation. The user scenario that was designed in close collaboration with the care providers (according to the principles of the participative design methodology $(19,20))$ targeted the reinforcement of the communication between the elderly residents and the visitors (family \& friends) by bringing the world outside the residence closer and cherishing the memories. By means of the VPAN technology a specific TV channel was added to the TV-set of the elderly person which allowed to exchange messages and pictures within the community of friends and family. The results of this experiment indicated that the application could indeed enhance a closer contact between visitors and elderly persons. Most elderly persons prefer many short contact moments compared with few long visits. The fact that the VPAN system allowed feedback (the fact that the pictures/messages had been seen) was considered a motivation to add additional content to the system, hence improving the contact. During the actual test period, this improvement was most prominent and satisfying for the users when connecting the elderly person with the grandchildren; this observation did differ from the initial assumption that it would mainly improve the communication between the elder and the oldest child (primary caregiver).

Although the both the application experiment at the "OCMW-De Vijvers" organisation and the VPAN technology proved to be successful, an actual go-tomarket scenario has not yet been identified. The VPAN concept has also been used in other research projects such as Magnet and Deus.

\section{Business axis}

During the TranseCare project we analyzed the economic viability of eCare solutions. A multi-actor analysis was executed, calculating for each actor, depending on the roles they are fulfilling, their costs and benefits in a dynamic way. A detailed analysis of all roles required to offer eHomeCare services was elaborated. Several business models have been analyzed and compared, where different provider take up a dominant role in delivering eHomeCare services towards the patients. The results show for the health care actors (general practitioners, home care and retirement homes) social benefits, either in terms of new customers, time savings or serving the needs of customers. The largest components of the tariff paid by the patients include call centre and network connectivity costs. The eCare infrastructure would ideally be subsidized by the government to enhance the speedup of rollout of eHomeCare services. We concluded that all actors participating in the offering of the eHomeCare platform and services can benefit from the imple- 
mentation of the system, which is of great importance to help formulating a business model towards governmental agencies, insurance companies, health care providers or private investors as to how much and how soon to invest in eCare.

\subsection{Was the project continued?}

As both projects were funded out of the Flemish research funds, which stops at proof-of-concept phase, neither project was continued as a whole. However, several aspects did continue after the termination of the projects:

The exchange hospital - home care is succeeded and the project will continue. Started from some 'easy to implement' (but necessary) data to exchange, other data-exchange will follow. These phases however will proceed out of the context of a project. Because it is necessary, hospital and homecare will invest themselves.

The video communication device has been brought to the market as the "Xtramira" ${ }^{\mathrm{TM}}$ " and is actively being promoted by Televic Healthcare. At present the product has attracted the interest of the market, not only in the originally targeted homecare setting, but also in the residential elderly care market.

The video communication experiment has been taken up by a lead customer: OCMW-Kortrijk, which has evolved into a showcase on the living lab approach in Flanders (21). Furthermore this experience has been taken to the European level in the context of the Apollon (22) project where the solution will be brought to the living lab in Helsinki, Finland.

The socio-economic modelling has received much attention from generic industry platforms on eHealth. There it will be investigated to what extend these results can be used in future scenario's to transfer terms as "better quality of care" into objective numbers which can be used for policy decisions. 


\section{Internal influences on development and outcome}

\subsection{What requirements were expected from those involved in the project}

During both projects there has been a constant attention and continuous evolution with respect to the requirements. The white-book approach proved to be an essential tool to facilitate this process without ending up with projects where development is blocked due to constantly evolving requirements. During this process a number of "rules-of-thumb" were used as guidance:

- Focus on realistic goals: better less ambitious but possible than blue sky and nothing realized

- Don't forget the user requirements (compare to the technological); they need to be gathered for different elements within the communication network (alert system calls, multiplayer gaming, video chat application, ...)

- Manage expectations across disciplines during the entire project cycle: what is expected from healthcare and what is healthcare expecting from its technological partners?

More concrete the expectations in the projects can be summarize as follows:

- Healthcare is actively searching for technology working as an assistant of the nurse and/or as a facilitator for the elderly. As already mentioned the number of elderly is increasing faster that the number of nurses (see 2). Also, the elderly are more isolated in their homes since their social network is smaller. Hence the expectation of assistive technology. Healthcare expects a technology adapted to the user profile with an ease to use. Is the support of the technology matching the requirements of the user groups?

- The industry expects from the healthcare an actively participation with testing the applications, a constructive commenting and forwarding feedback concerning user experience and future expectation.

- The healthcare organizations are expected to actively search for test persons fulfilling the inclusion criteria and personnel.

\subsection{How and due to which causes did those requirements change during the process}

The very nature of both the COPLINTHO and the TranseCare project implied a change in requirements during the process. Indeed, since both projects had the 
combined ambition to advance user centred design of ICT services for home care as well as advancing the actual ICT technology at the same time, a conflict situation arises. This conflict can be illustrated by the fact that traditionally technical requirements are being drafted when user information is available while at the same time the user scenarios have to be created according to the possibilities of the technology envisaged. The mere fact that neither the technological development nor the user research was only finalized at the end of the projects implied a change in requirements.

Additionally the requirements are also influenced by the actual availability of test persons. Especially elderly homecare settings have to cope with difficulties due to ageing, increasing illness or worse. Based on the initial requirements, the healthcare organizations searched for test persons between their patients. However the enrolment criteria were very challenging. Is the user profile fitting the requirements of the application? Is this application suitable for elderly with dementia, or mentally impaired persons? Or everybody? Should the application be simplified? Do the users need a more simplified application?

The white book approach proved to be an excellent tool to manage this variability. It allowed to "softly" fix both user requirements and technological requirements at an early stage while providing sufficient room for adaptation as technological risks turned out to be not feasible or user selection to complex.

\section{External influences on development and outcome}

\subsection{Did economic aspects play a role in the development and outcome of your project?}

As with any project, economics play a role in the development and outcome of the project. Both the COPLINTHO and the TranseCare projects had approved funding, however the funding level was different for academic, non-profit and profit sector partners (ranging from $100 \%$ to $0 \%$ funding). The fact that the funding mechanism was available proved to bootstrap this multidisciplinary project approach. Without it, there would have been little chance that such a diverse multidisciplinary team would be created in the Flemish context.

The economic aspects of the solution were explicitly taken into account during the projects. Not only the possible cost factor of the resulting solution but also the search for business models that can enable the eHomecare market to develop. Indeed, in the end the only economic aspect that is perceived by the end user is the price for that eHomecare user. We see that the elderly users of today are willing to 
use ICT-applications and that their acceptation is high. However, we should take into account that this is also only with a limited cost for these applications. (23)

The search for a viable cost/benefit model for all actors involved to support and fund large eHomeCare projects will be crucial for the development of the Flemish market.

\subsection{How did the project development and outcome affect the partners of the project, both individually and as a consortium?}

The vision of industry and healthcare is traditionally opposite oriented; healthcare practices start typically from a user perspective with "watching" the patient; what are the patients needs; which type of technology is accessible (use \& cost) for our target population? This is rarely the case for the ICT industry, which is typically oriented towards technology driven developments. Combined with the fact that ICT developers are typically young of age, the end result is often that product ideas are disconnected with actual situations and the target user group of elderly persons.

During this multidisciplinary collaboration a change in mind set was established on both sides:

The ICT-organisations approached care

By collaborating in the multidisciplinary team, the ICT organizations gained knowledge and better understanding of the actual environment and situation of the elderly persons. Examples can be found in the knowledge that retired persons are not very often confronted with new types of technology (hence have less adaptivity skills), that they have a different ICT-intuition and handling skills (e.g. handeye coordination for navigation or use of the remote control, as illustrated in figure 2) which gives the term "usable" a whole new dimension. 


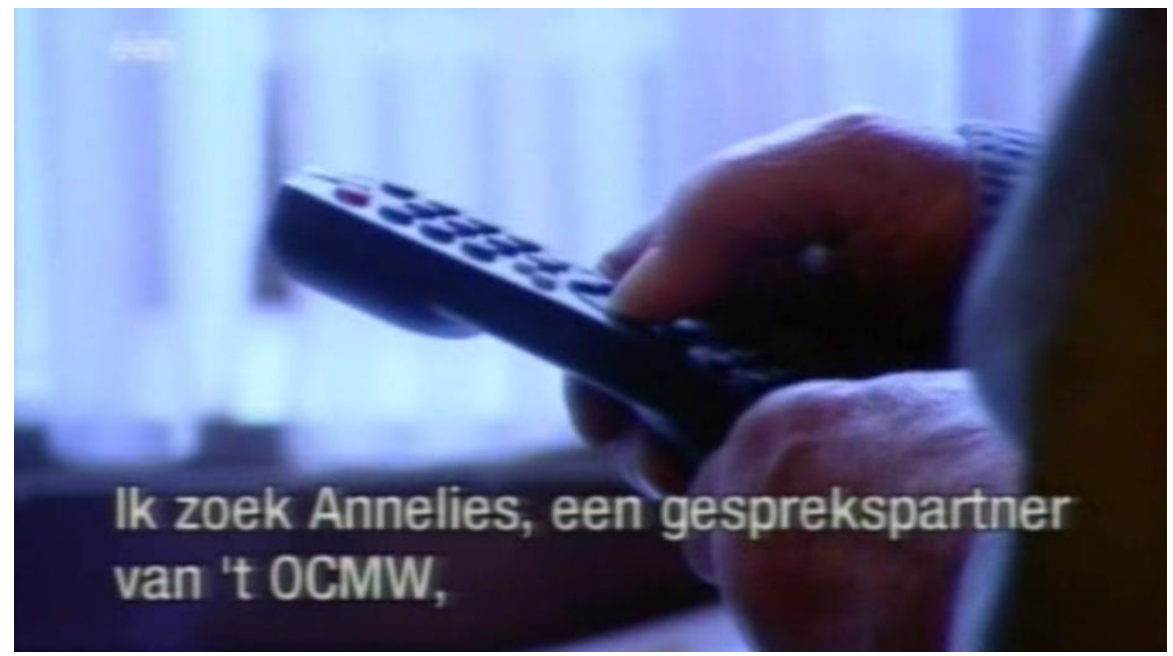

Figure 2: example of remote control use by elderly: typically two hands

The care partners approached the ICT industry

By having to explain the needs and being involved in the development process, the healthcare organizations also gained knowledge and a better understanding of the industry driving forces. Examples of this process can be found in the increase in confidence that technology can be used when adapted to the user, the joined search for business models instead of the strict need to have cheap (free) technology for the end user.

The result can be summarized in the fact that the healthcare needs to gain confidence in the use of the technology and the application, the ICT organizations need to gain confidence in the fact that adapting to the (end) user environment can result into business and both sides need to gain confidence that the elderly can (and want to) participate and use the technology.

\subsection{Did legal issues affect the development and outcome of the project?}

Several legal issues were investigated during the projects, mainly focusing on privacy issues of all actors involved.

A first result of this study was to implement a specific feature in the video communication device (Xtramira ${ }^{\mathrm{TM}}$ ) that enabled the user to remain in full control of his privacy with respect to activating the local camera connected to the Xtramira device. In case of alarm condition, the local camera is automatically activated. A practice that fully conforms with legal conditions. 
A second result of the legal study was that the field trial had to be limited to test with a system that only used a single camera for communication purposes. Hence, the scenario which encompassed multiple cameras that allow the desk caregiver to search for the elderly person by activating the cameras from a distance was demonstrated in realistic conditions but not actually used with end users during field trial.

\subsection{Were regional aspects involved in the development and outcome of the project?}

As the use of ICT does not imply strict regional limitations to the deployment of the eHomecare service, regionality was not taken into account for the field trials in the COPLINTHO project. Although this demonstrated the possibility to roll out ICT based eHomecare services over a large territory, it should be considered that healthcare always has a local/regional component that needs to be taken into account. Indeed, providing physical home care implies a regional organization, hence the current a regional structuring of care coordination.

During the TranseCare project, a more regional approach was taken, which not only proved to be more close to existing care structures but also proved to be more practical to cope with technical field trial issues.

If ICT is to be successful in the home care domain, it has to respect that existing organizational structure, i.e. supporting the home care rather than implying a new organizational model. When ICT will prove to support and enable additional efficiencies, the care organizations will gradually and automatically adapt their structures to a larger regional scale.

\section{User aspects}

\subsection{Did (possible) implementation of your project involve educational challenges? If so, how were they (to be) met?}

A first educational challenge was met during the early stages of the projects. Indeed the multidisciplinary team combined members with very different backgrounds and all were confronted with specific terminology: not only the traditionally difficult technological terms and acronyms but equally confronting were the specific healthcare terms and scales. A nice example of this conflict in terms can be found in the term "protocol"; used in a technological context it designates a method by which different devices communicate; in the healthcare context it is be- 
ing used to designate specific steps in a treatment or care process. Each time new members were added to the multidisciplinary team, specific time had to be spent in order to close the gap and reach a sufficient level of understanding.

Educational challenges were also encountered at the level of the test persons (both elders and nurses): research has shown that the current group of elderly is willing to accept or adopt new technology if this is improving their quality of life. However, what they do not want, is to lose control of their choices (freedom) or their privacy. The TranseCare project involved a camera, internet (i.e. equipment that was introduced into the home by the project) and the use of their own television (already present).

The educational challenges encountered by the healthcare organization were specifically related to the approach to enrol test persons into the project. The enrolment procedure used had to be adapted such that it did put more emphasis on the unchanging privacy and the personal freedom of the test person to accept or decline a call. The need to learn more about the technology behind the application itself was not present, so this was not extensively explained.

A first contact to enrol a patient was by phone. During this phone call we checked the interest towards the project. We noticed a lot of "scary" persons that seemed to have reservations towards the use of technology, which could be caused by the mental state of the person contacted. However, the initial phone call starting with the message "This is the alarm central speaking..." was too invasive and confusing for the person being called. To keep the link between the alarm central and the project, it was decided to call the primary caregivers instead and ask whether his/her parent/family member/neighbour could be interested. If the primary caregiver gave a positive indication, the question was asked to contact the elderly person and to discuss the basics of the project. If interest remained, a personal contact between the elderly person and healthcare was organised. This change in approach was vital to convince possible test persons to join the test program.

For future cases, it might be useful to search for test patients by contacting the leading nurses of different departments instead of only contacting one as was the case in the projects. The holistic approach to care of the homecare nurse is often sufficient to get an estimate on the capabilities and/or interest of the patient.

We also noticed that it usually were the children of the contacted person that were enthusiast about the technology and were trying to convince their family member.

Another, long term, educational change should be made to the training of the nurses. At present, most nurses are confronted with this type of technology for the first time during their actual working situation. It should be more efficient to inform them earlier, e.g. during their school education period. The sooner they realize what technology can bring to them, their patients and his/her environment, the easier it will be to convince them to try the technology themselves and to support their patients. 


\subsection{Were user satisfaction and usability part of the project's goal and how?}

In eHomecare user satisfaction is an important means to gain the confidence of the users. As mentioned before, research has shown that the "upcoming" baby boomers are very interested in technology. However, the research also concluded that these potential users want to use technology if their freedom and privacy is guaranteed. The current elderly population (prior to the baby boomers, also called the pre-war generation) has grown up with little or no technology adherence and with a "natural" fear for this technology. Therefore this pre-war group needs especially the product satisfaction to gain confidence.

Hence, both user satisfaction and usability were part of the projects from the start, however the focus was more oriented toward user centric design and evaluation by user rather than big statistical user satisfaction studies. For each demonstrator that was build, specific attention was given during the design and development process to realize a solution that could meet the user expectations and could also be used by the non-tech-savvy end users. Already early in the development process user feedback was achieved based on low fidelity prototypes (mock ups, paper printouts of the screens, etc.). The usability of the first operational high fidelity prototypes was evaluated during a lab test under controlled conditions with test users in observation rooms. During the field trials, a similar user evaluation was executed; however extensive observations are less obvious in the field. Hence the feedback was triggered using in depth interviewing techniques in order to get insight in the user motivation.

The result of this approach was a successful evaluation of an easy to use central desk application as well as the easy to use interface of the Xtramira ${ }^{\mathrm{TM}}$ device. Actual observation tests with different test persons with ages ranging from 57 to 75 clearly showed that $90 \%$ of the test persons could execute the test sequences based on the embedded instructions, without any additional training.

\subsection{Did user aspects influence the final outcome of the project?}

During both projects user aspects were constantly balanced with technological requirements in order to create the multidisciplinary solutions which the projects targeted. In order to gain insight in some specific user aspects, field observations were organized to study comparable products to create a funded understanding of the user context. More specifically a study of the knowledge of the symbols used on the buttons of phones and remote controls was performed in order to provide recommendations for the development of the Xtramira ${ }^{\mathrm{TM}}$ symbolic language and remote control. 
As both projects also used cameras at the user's premises, specific attention was given to study the reactions of the test persons with respect to this feature. The research showed that especially the presence of the camera was disturbing, resulting in strange attempts to safeguard their privacy by covering the camera, as shown in the figure below. The fear of having someone to see their homes trough the camera when they do not know and losing control is for the contacted persons too high to accept/adopt the technology. Therefore, the final design of the Xtramira ${ }^{\mathrm{TM}}$ was adapted to include a very small camera in order to easy the user in getting used to the system without being constantly focused on the large lens in the living room. Additionally the application interface of the Xtramira ${ }^{\mathrm{TM}}$ was modified in such way that it is obvious and intuitively clear to the end user when the image is being transmitted to the other side.

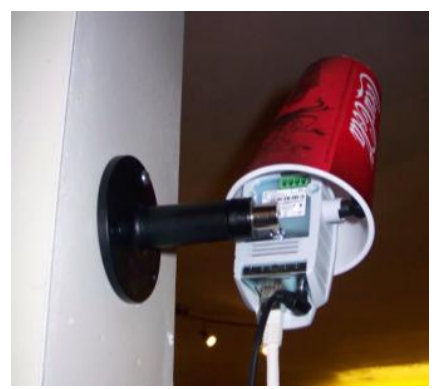

Figure: creative user methods to safeguard privacy

\subsection{What were the reactions from users on your project?}

To fulfil the inclusion criteria, the test user had to be a patient of the "Wit-Gele Kruis" organisation in the Limburg-province, he/she should have on the KatzWeckx scale a mobility score of 2 or more, toilet visit of 2 or more and should live alone. These criteria are not easy in that way that most of these patients have troubles with using the technology themselves. When contacting the caregivers to check for their interest, the most common comments were "this is nothing for my parent. He/she is too scared and too confused to use this application." "It will disturb her/him too much." "She/he is not using the phone, this will be way too difficult for her/him". One patient that fulfilled the enrolment criteria gave informed consent to participate to this project. She and her family were very enthusiast to use this application. After installing the application at her home and solving the first technical challenges, it became clear that she was too nervous to use the system. It was difficult for her to understand how to accept or start a call. The mental state of the user worsened faster than expected. The system was removed when it was too disturbing and confusing with respect to the mental state of the test person. 
To find patients, a call and visit were made to a department of the "Wit-Gele kruis"-organisation. The leading nurse checked the general patient list and discussed with the nurses of that department on her weekly meetings the requirements. Every nurse checked between his/her patients to check whether there was a patient fulfilling the criteria. On general, these nurses reacted very positive on the new technology although they were very scared as well to use the application. They were scared to "break" the system and that the help-line would not be enough to solve an issue when it would occur. Some of them even "hoped" that they would not have a patient, so they did not have to manage the technology. The nurse of the actual test person was also worried, but very enthusiastic as well. She managed very well to start the application, comfort the patient and help her to sort her mind when the test user was disturbed. The nurse mentioned also that the sound should be easier adjustable for the hearing impaired.

For the alarm central, that receives all calls of the personal alarm system, it was not difficult to use the system, but still too disturbing since the alarm was used together with the usual alarm system. Using both systems next to each other could cause an overload of work.

The test users with respect to the social contact scenarios were very positive as the solution was perceived as a decrease of the social isolation. This effect was observed at the side of the elderly person but also at the side of the children and grandchildren. In one specific case, the use of the system even realized the granddaughter to get interested into details of the earlier life experiences of her grandparent, an aspect which was unknown and had not been addressed earlier by the granddaughter.

Most test users were also very positive with the non-stigmatic combination of social contact communication and alarming device that was enabled by the Xtramira $^{\mathrm{TM}}$ solution.

\section{General aspects}

\subsection{Did your project have an effect on matters like efficiency, quality of labour, quality of care and quality of life?}

Neither the COPLINTHO project nor the TranseCare project had specific tasks during the project to objectively evaluate matters such as efficiency, quality of labour, care and life. However, based on the results of the field trials and the positive responses of the different users, positive effects are to be expected. In case the project results would be deployed on full scale, they would certainly increase the quality of labour for the central desk nurses at the alarm central organization. The same result is expected for the caregivers as the system allows for a more person- 
alized touch and the time gain due to video communication will normally be converted into additional time spent with the patient. During the project a family caregiver mentioned a similar effect, i.e. spending more time on the actual communication rather that a very short visit due to travel times.

As the video system also allows for a better understanding and insight in the patients situation and environment, it is easier to quickly grasp the seriousness level of an alarm (or the lack of in case of false alarms). This results in a lower dispatching rate in case of false alarm, hence resulting in a better use of the care staff.

As indicated before, the increase in social contacts and the breaching of the sense of social isolation (made possible through the solutions created in the projects) resulted in a sense of better quality of life for the users.

\subsection{What kind of dissemination strategy did you develop / use?}

During the project, several dissemination strategies were used. Besides the traditional publications along the multidisciplinary domains, specific workshops and seminars were organized. The purpose of these events was twofold: dissemination of the results and creating awareness in the homecare sector, as well as using the audience as sparring partner to get feedback on the approach taken. This effect was most prominently present in the workshops relating to the business modelling.

Additionally a portable version of the demonstration set up was also shown at several local care events, hence creating the awareness that technology can provide a benefit to the homecare sector.

Finally a short movie was made at the end of both the COPLINTHO and the TranseCare project. Each time the movie tells the story from the viewpoint of the end user (i.e. patient, nurse, family) and demonstrates how the solution and technology can support the healthcare process.

Although creating such a movie was a tedious task and involved skills not present in the project consortium, the reactions of the different audiences proved that it is very effective in communicating the results of multidisciplinary projects which tend to be complex to explain. It even proved to be an effective marketing tool during the early market introduction days of the Xtramira ${ }^{\mathrm{TM}}$ product.

\section{Conclusion}

The results of the multidisciplinary projects COPLINTHO and TranseCare clearly illustrate that this approach can be very successful to create solutions that bridge the gap between the high tech world and the homecare world. The methods developed and used during the projects prove that it is indeed possible for tech- 
savvy and non-tech team members to learn to understand each other and to collaborate in order to design solutions adapted to the end users.

Although there are still hurdles to take in order to fully bring the resulting solutions up to a full scale deployment, the results clearly illustrate that technology in homecare belongs to the near future. These solutions give opportunities for the elderly at home to broaden their social network; in a time where families become smaller, and people become more individualistic, video communication opens a window to the world and can provide access to a higher quality of life.

\section{Reference}

Acknowlegment: The results presented in this chapter were based on the successful collaboration of the COPLINTHO and TranseCare partners: IBBTUGent/IBCN, IBBT- UGent/MIG-RAMIT, IBBT-V.U.B./SMIT, IBBTK.U.Leuven/CUO, IBBT-K.U.Leuven/ICRI， IBBT-UHasselt/EDM，Wit-Gele kruis VZW, Televic N.V., Medibridge N.V., Custodix N.V., Androme N.V., AZSt. Elizabeth, UZ Gent, In-Ham VZW., Solidariteit voor het Gezin VZW.; both projects were partially funded by IBBT and IWT.

${ }^{1}$ COPLNTHO acronym stands for: "Innovative Communication Platforms for Interactive eHomeCare".

2 TranseCare acronym stands for "Transparant ICT platforms for eCare"

${ }^{3}$ Centraal scenario van de vooruitzichten 1995-2050, Nationaal instituut voor de statistiek, Federaal Planbureau, 1996

${ }^{4}$ PAS: personal alarm service, an existing service that allows the user to activate an alarm and set up a phone communication with a central desk nurse which can dispatch help if needed.

5 Yogesan K, Bos L, Brett P, Gibbons M.C., editors. Handbook of digital Homecare, Springer, 2009, "A Multi-disciplinary Approach towards the Design and Developmen ot Value ${ }^{+}$eHomeCare Services"

${ }^{6}$ Cooper, A. "The inmates are Runing the Asylum", SAMS, 1999

${ }^{7}$ Caroll John, M. Making use : Scenario-based design of Human-Computer interactions, MIT press, Cambridge, 2000

${ }^{8}$ Pruitt John, Adlin Tamara. The Persona Lifecycle: Keeping People in Mind Throughout Product Design. Morgan Kaufmann, 2006

${ }^{9}$ www.xtramira.com

${ }^{10}$ Hoebeke J., "Adaptive Ad Hoc Routing and Its Application to Virtual Private Ad Hoc Networks”, Phd, ISBN 978-90-8578-172-1, NUR 986, 2007

${ }^{11}$ Arnaert A. Tele-nursing for the elderly, the case of videotelephone care, explorative study, PhD, K.U.Leuven, 2001

${ }^{12}$ Marilynne A. Hebert, Barbara Korabek (2004). "Stakeholder Readiness for Telehomecare: Implications for Implementation", Telemedicine Journal and eHealth. March 2004:, 85-92 
${ }^{13}$ María E. Dávalos, Michael T. French, Anne E. Burdick, Scott C. Simmons (2009). "Economic Evaluation of Telemedicine: Review of the Literature and Research Guidelines for Benefit-Cost Analysis". Telemedicine and e-Health, December 2009: 933-948

${ }^{14}$ Stephanie Vergara Rojas, Marie-Pierre Gagnon (2008). "A Systematic Review of the Key Indicators for Assessing Telehomecare Cost-Effectiveness". Telemedicine and e-Health, November 2008: 896-904.

${ }^{15}$ Kathryn H. Dansky, Liisa Palmer, Dennis Shea, Kathryn H. Bowles (2001). "Cost Analysis of Telehomecare". Telemedicine Journal and e-Health. September 2001: 225-232

${ }^{16}$ Van Ooteghem Jan. "Multi-Actor Analysis for Identifying the Economic Viability of eCare Solutions", to be presented at Medicine 2.0 conference, November 29-30, 2010, Maastricht, the Netherlands

${ }^{17}$ Van Ooteghem Jan, Ackaert Ann, Verbrugge Sofie, Colle Didier, Pickavet Mario, Demeester Piet. "Economic viability of eCare solutions", to be published in the proceedings of eHealth 2010 conference, December 13-15, 2010, Casablanca, Morocco

${ }^{18}$ Onderzoek uitgevoerd door het Centrum voor Publieksonderzoek van de KU Leuven, in opdracht van het Vlaams Instituut voor Wetenschappelijk en Technologisch Aspectenonderzoek (viWTA): Kleurrijk vlaanderen kleurt grijs, eindrapport, Leuven, juli 2004

${ }_{19}$ Bijker, W.E. (1987). The social construction of technological systems; New directions in the sociology and history of technology. Cambridge AM: MIT Press.Bijker, 1987

${ }^{20}$ Sleeswijk Visser, F., Stappers, P. J., Van der Lugt, R., Sanders, E. B.-N. (2005). Contextmapping: experiences from practice. CoDesign: International Journal of CoCreation in Design and the Arts, 1(2), 1-30

${ }^{21}$ NEXTlab project, funded by IWT (2010-2012)

${ }^{22}$ Apollon, FP7 project, www-apollon-pilot.eu (2009-2011)

${ }^{23}$ Telecare voor ouderen: Socio-economische analyse van het gebruik van video-telefonie binnen de ouderenzorg / Peetermans Annick, Hedebouw Georges, Pacolet Jozef, Devoldere Paul, D'Haene Filip, Pouillie Roland, et al. Leuven: Hogeschool instituut voor de arbeid, 2004 